\title{
Microbial imprints as forensic tools in food production
}

\author{
B. F. Smets, W. H. Verstraete and S. D. Siciliano \\ Laboratory of Microbial Ecology and Technology (LabMET), Ghent University, Belgium
}

Eukaryotes exist in close association with a wide variety of microorganisms. Some of these microorganisms are pathogenic or beneficial but the vast majority have little.discernable impact on eukaryote functioning. These microbes typically colonize portions of eukaryotes that interact with the surrounding environment. The rhizosphere, the phyllosphere and the root interior are examples of interphases in which plants either alter their environment, take up nutrients or respire. These interphases are all heavily colonized by bacteria. Similarly, animals have bacteria colonizing their epidermis, gastrointestinal tracts and respiratory organs. Because these microorganisms are in contact with the environment as well as the host, it is likely that the composition of the microbial community at this interphase is jointly influenced by the host and the environment.

There are a number of interacting host-related and environmental factors that influence the composition of the associated microbial community. For example, the health and genome of the eukaryal host can alter the physical structure and chemical conditions of the environment that the bacteria colonize. Similarly, the presence of toxicants in the air or water phase that the host exchanges with the environment can influence the structure of the associated microbial community. Recent advances in molecular ecological techniques permit us to directly inspect the make-up of the microbial community in association with eukaryal hosts. As a result, a wealth of descriptive studies on these microbial communities has been initiated employing a wide variety of refined molecular techniques. What benefit can be reaped from this information? We hypothesize that the microbial signature of a eukaryal host can identify from which environment the host has originated as well as the host health status. Thus, we propose that the information on eukaryote-associated microbial community composition be used to infer the site of origin and the cultivation conditions of the eukaryal host.

The most obvious application is in the food industry. The ability to identify whether a crop is transgenic or not, or to identify whether a crop in a particular soil has been grown under organic or conventional conditions is of immense interest to the public at large. Regulators and food inspectors have a difficult time tracing the origin of food products. Recent work has identified that microbial communities of transgenic crops differ from their parent stock and that the microbial community present inside the plant is highly sensitive to xenobiotic compounds present in the soil. These microbial communities are an integral part of the food itself and, therefore, one can still assess the associated microbial community long after the harvest. To date, the food industry has concentrated on using this information to insure the absence of pathogens from the food supply chain. We suggest that, in the near future, assays will be available that can identify the management conditions under which a food has been grown and downstream processed and that might even localize the food's geographical origin.

To accomplish this goal of using eukaryote-associated microbial imprints as forensic tools, we must continue to develop rapid microbial community typing techniques as well as data reduction techniques to yield new metrics that express microbial community characteristics in a simple manner. Besides such technical advances, there are important knowledge gaps that need to be filled before we can use microbial imprints as a tracer of food source and quality. We need to understand what signatures in microbial community composition are dynamic or persistent, the time scale of change and the impact of transient management practices. We need to assess what information content present in the microbial community composition is indicative of past events. Such conditioning effect is quite commonly seen in macroecology but has not yet been demonstrated, except for extreme events, in microbial ecosystems. Microbial ecology has been wrestling with techniques ever since the Winogradsky column was developed. We enter a new era, one in which advances are not dependent on techniques, but one where we can begin to explore how fundamental molecular processes occurring in the environment can be harnessed for the public good. Prime amongst these goals should be attempts to ensure the quality and traceability of our food. 